\title{
TECHNOLOGY FOR AUTOMATIC SOIL SAMPLING BY MANiPUlators MOUNTED ON UNDERWATER VEHICLES
}

\author{
Vladimir Filaretov, Alexander Konoplin, Nikita Konoplin \& Georgy Gorbachev
}
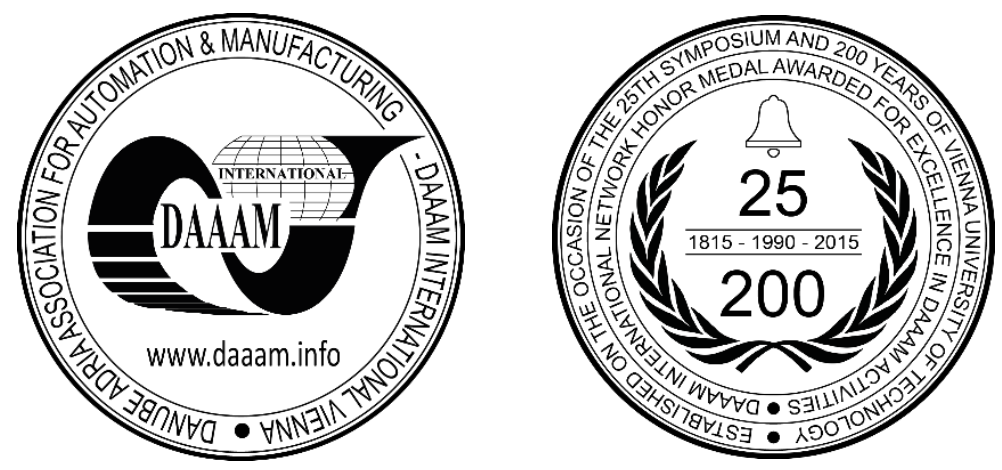

This Publication has to be referred as: Filaretov, V[ladimir] F[edorovich]; Konoplin, A[lexander]; Konoplin, N[ikita] \& Gorbachev, G[eorgy] (2017). Technology for Automatic Soil Sampling by Manipulators Mounted on Underwater Vehicles, Proceedings of the 28th DAAAM International Symposium, pp.0679-0684, B. Katalinic (Ed.), Published by DAAAM International, ISBN 978-3-902734-11-2, ISSN 1726-9679, Vienna, Austria

DOI: $10.2507 / 28$ th.daaam.proceedings.095

\begin{abstract}
The work proposes the technology for an automatic implementation of the soil sampling by means of underwater multilink manipulators which are attached to submergible autonomous and remotely controlled vehicles. This technology includes the strategy of the safe approaching of a vehicle to a sea bottom surface, and also the formation of manipulator trajectories for the efficient soil sampling by hermetically sealed tubes and soil scoops. The results of sea tests are presented for the study of the working capacity of the proposed technology.
\end{abstract}

Keywords: underwater vehicle; multilink manipulator; automatic mode; soil sampling; trajectory planning

\section{Introduction}

Today, the world Ocean exploration is impossible without performing of research operations by means of underwater vehicles (UV). Up to now, the most of manipulation operations are performed with the help of remotely operated UV equipped with multilink manipulators (MM). Specially trained operators remotely operate the UV with MM from the laboratory on a carrier vessel in the process of the underwater operation performing. However, the efficiency of these operations is highly dependent on the weather conditions, since the presence of a flexible cable of a remotely operated UV does not allow to performs precisely manipulation operations under conditions of significant displacements of the carrier vessel. Moreover, the use of a remotely operated UV in the conditions of the northern seas for most of the year is impossible due to the presence of seas ice covers. Therefore, it is necessary to develop new modern control systems and technology for the UVs and its MMs to perform manipulation operations in an automatic mode. This will allow to use autonomous UVs equipped with MMs for the implementation of underwater operations independent of the weather conditions and even in conditions of seas ice covers.

The systems that provide accurately automatic movement of the UV along complex spatial trajectories $[1,2]$ are already developed. For the taking into account negative angular displacements of the UV under the influence of the grasped objects and probes the methods $[3,4]$ are developed. The technologies for high-precision automatic stabilization of the UV in the hang mode [5-8] are already created. These stabilization systems allow to perform manipulation 
operations without additional mechanical devices for the UV rigid fixation near work objects and exclude the introducing of disturbances into bottom layers of water.

There are the known cases of using the autonomous UV for the performing of the simplest manipulation operations in the automatic mode $[9,10]$. In these cases, a stereo vision system and sophisticated image processing algorithms for detecting objects, which have a previously known form, are used to determine locations and spatial orientations of working objects with respect to the UV. Furthermore, a complexity of implementation of the MM automatic control is also due to high complexity of the MM working tools trajectories planning in conditions of variability and uncertainty of the environment.

The approach for automatic implementation of manipulative operations near the sea bottom in hang mode of UV was proposed in the previous paper [11] of the authors. The approach provides the definition of a sea bottom surface position relative to the UV using standard hydroacoustic onboard sensors, and allows to form trajectories of MM motions so that the MM provides minimum possible force and torque effects on the UV during the operations.

For the automatic performing of manipulation operations by UV it is necessary to develop a method that estimates a complexity of the sea bottom relief in a working area in a process of UV approaching to the bottom surface, as well as makes decisions about suitability of this sea bottom relief for the failsafe implementation of manipulation tasks. It is also necessary to rotate the UV towards the rise of the seabed surface [11]. This UV orientation will allow to take the most convenient position for the performing of the manipulation operation. Furthermore, a hovering position for the automatic UV stabilization near a bottom should be calculated automatically. In this place, a MM workspace should allow to properly take a sample of soil, avoiding of collision with a ground and resuspension of a bottom water layer. Thus, the trajectory of MM working tools should be formed taking into account dimensions of the sampler, characteristics of a MM workspace and with an information about continuously refines model of the sea bottom surface.

As a result, the process of automatic performing of soil sampling by UV has to be presented as sequence of the next actions (see Fig. 1):

- The submerging of the UV while the distance to the bottom will allow to estimate a complexity of the sea bottom relief in a working area.

- The estimation of the sea bottom relief complexity in a working area.

- The UV rotation toward the rise of the sea bottom surface.

- The approaching of the UV to the bottom surface, until the surface is in the working area of MM.

- The performing of manipulation operation.

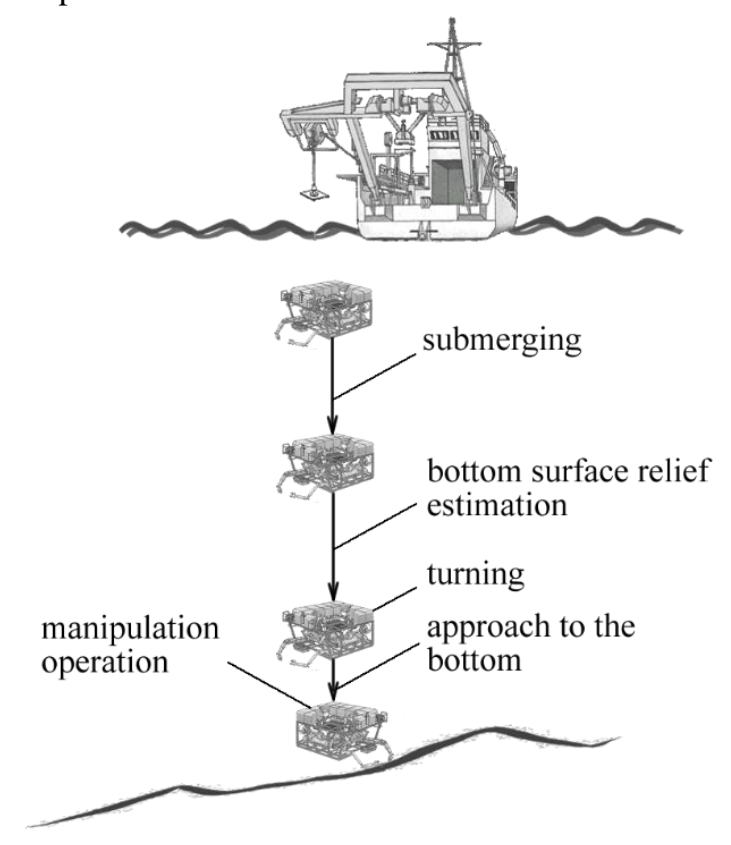

Fig. 1. The automatic performing of manipulation operation as sequence of the actions

\section{Features of the UV approaching to the bottom surface}

In the process of the UV submerging to the sea bottom surface, the determination of the space position of this surface relative of the vehicle can be obtained by the method [11]. According to this method, the bottom surface is described in the rigidly connected with the UV right rectangular coordinate system $X Y Z$ by the equation:

$$
A^{*} x+B^{*} y-z+C^{*}=0
$$


where $x, y, z$ are the variables; $A^{*}, B^{*}, C^{*}$ are the constant coefficients which are simultaneously not equal to zero. These coefficients, updated with each measurement of the Doppler lag, can be calculated by the equation:

$$
\begin{aligned}
A^{*}= & \frac{n \sum_{i=1}^{n} z_{i} x_{i} \sum_{i=1}^{n} y_{i}^{2}+\sum_{i=1}^{n} x_{i} y_{i} \sum_{i=1}^{n} y_{i} \sum_{i=1}^{n} z_{i}+\sum_{i=1}^{n} z_{i} y_{i} \sum_{i=1}^{n} y_{i} \sum_{i=1}^{n} x_{i}-\sum_{i=1}^{n} z_{i} \sum_{i=1}^{n} y_{i}^{2} \sum_{i=1}^{n} x_{i}-n \sum_{i=1}^{n} z_{i} y_{i} \sum_{i=1}^{n} x_{i} y_{i}-\sum_{i=1}^{n} z_{i} x_{i}\left(\sum_{i=1}^{n} y_{i}\right)^{2}}{n \sum_{i=1}^{n} x_{i}^{2} \sum_{i=1}^{n} y_{i}^{2}+2 \sum_{i=1}^{n} x_{i} y_{i} \sum_{i=1}^{n} y_{i} \sum_{i=1}^{n} x_{i}-\left(\sum_{i=1}^{n} x_{i}\right)^{2} \sum_{i=1}^{n} y_{i}^{2}-n\left(\sum_{i=1}^{n} x_{i} y_{i}\right)^{2}-\left(\sum_{i=1}^{n} y_{i}\right)^{n} \sum_{i=1}^{2} x_{i}^{2}}, \\
B^{*}= & \frac{\left.\left.n \sum_{i=1}^{n} x_{i}^{2} \sum_{i=1}^{n} z_{i} y_{i}+\sum_{i=1}^{n} z_{i} x_{i} \sum_{i=1}^{n} y_{i} \sum_{i=1}^{n} x_{i}+\sum_{i=1}^{n} x_{i} y_{i} \sum_{i=1}^{n} z_{i} \sum_{i=1}^{n} x_{i} x_{i}^{2} \sum_{i=1}^{n} y_{i}^{2}+2 \sum_{i=1}^{n} x_{i}\right)_{i} y_{i} \sum_{i=1}^{n} y_{i} z_{i} y_{i}^{n} x_{i}-\left(\sum_{i=1}^{n} x_{i}\right)^{2} \sum_{i=1}^{n} y_{i}^{2} x_{i} y_{i} \sum_{i=1}^{n} z_{i} x_{i}-\sum_{i=1}^{n} x_{i}^{2} \sum_{i=1}^{n} y_{i} \sum_{i=1}^{n} z_{i} x_{i} y_{i}\right)^{2}-\left(\sum_{i=1}^{n} y_{i}\right)^{2} \sum_{i=1}^{n} x_{i}^{2}}{n \sum_{i=1}^{n} x_{i}^{2} \sum_{i=1}^{n} y_{i}^{2}+2 \sum_{i=1}^{n} x_{i} y_{i} \sum_{i=1}^{n} y_{i} \sum_{i=1}^{n} x_{i}-\left(\sum_{i=1}^{n} x_{i}\right)^{2} \sum_{i=1}^{n} y_{i}^{2}-n\left(\sum_{i=1}^{n} x_{i} y_{i}\right)^{2}-\left(\sum_{i=1}^{n} y_{i}\right)^{2} \sum_{i=1}^{n} x_{i}^{2}}
\end{aligned}
$$

where $x_{i}, y_{i}$ and $z_{i}, i=\overline{(1, n)}$ are coordinates of points of the array of $n$ points which are formed in the coordinate system $X Y Z$ by the Doppler lag. These points belong to a sea bottom surface.

Equation (1) can be written in the normal form [12] by multiplying its left and right parts on the normalizing factor $\operatorname{sign}\left(C^{*}\right) \frac{1}{\sqrt{A^{* 2}+B^{* 2}+1}}$ :

$$
A_{n} x+B_{n} y+C_{n} z=p
$$

where $A_{n}=\operatorname{sign}\left(C^{*}\right) \frac{A^{*}}{\sqrt{A^{* 2}+B^{* 2}+1}}, \quad B_{n}=\operatorname{sign}\left(C^{*}\right) \frac{B^{*}}{\sqrt{A^{* 2}+B^{* 2}+1}}, \quad C_{n}=\operatorname{sign}\left(C^{*}\right) \frac{-1}{\sqrt{A^{* 2}+B^{* 2}+1}}$ are the direction cosines of the unit vector $\bar{n}=\left[A_{n}, B_{n}, C_{n}\right]^{\mathrm{T}} \in R^{3}$, which defines the shifting direction of the bottom surface plane from the origin of the coordinate system $X Y Z$ at a distance $p=\operatorname{sign}\left(C^{*}\right) \frac{C^{*}}{\sqrt{A^{* 2}+B^{* 2}+1}}$.

At the stage of the UV submerging, this UV has to evaluate the suitability of the surface relief in a working area. It is necessary for the failsafe performing of manipulative tasks. The sea bottom area, covered by Doppler lag rays, is decreasing in the process of the UV diving. And the coefficients of the equation (2) are also changed together with this area. Experimental investigation shown that the evaluation of a value of these changes in a limited working area should be performed with a fixed heading angle of the UV. The measurements of these changes start when the condition $p \leq 10 L$ is satisfied, where $L$ is the maximum possible distance between the UV buoyancy center and the MM working tool.

The vector $\bar{n}_{k}=\left[A_{n k}, B_{n k}, C_{n k}\right]^{\mathrm{T}} \in R^{3}$ determines the bottom plane (2) spatial orientation in the coordinate system $X Y Z$ for the $k$-th measurements of all lag antennas, $k=\overline{(1, r)}$, where $r$ is the number of measurements. Evaluation of the bottom relief complexity can be based on the measuring of changes of angles $\alpha_{k}=\arccos \left(A_{n k}\right), \beta_{k}=\arccos \left(B_{n k}\right)$ and $\gamma_{k}=\arccos \left(C_{n k}\right)$, which are formed by the vector $\bar{n}_{k}$ with the respective axes of the coordinate system $X Y Z$. For all $r$ measurements while the UV dives with the stabilized heading angle, the test results shown that for the suitable working area surface (without major irregularities) the following conditions are usually satisfied:

$$
\begin{aligned}
& \alpha_{k \max }-\alpha_{k \min } \leq 15^{\circ}, \\
& \beta_{k \max }-\beta_{k \min } \leq 15^{\circ}, \\
& \gamma_{k \max }-\gamma_{k \text { min }} \leq 15^{\circ} .
\end{aligned}
$$

The UV submerging must be terminated if at least one of the conditions (3)-(5) are not satisfied. 
As it is noted in [11], if the UV is near the bottom, the UV longitudinal axis should be directed to the rise of the plane (2), because a workspace of the MM is located in the front semisphere of this vehicle. This UV orientation allows to improve visibility of the working area and preserve the biggest workspace of the MM. As a result, when the conditions (3)-(5) are satisfied and the UV is approached to the bottom surface to a distance $p \leq 2 L$, the UV should terminate its motion and turns to the rise of the bottom surface [11].

\section{Features of performing of soil sampling}

Place of the UV stabilization in the hovering mode [5 - 8] should be sufficiently close to a bottom surface for the soil sampling. But the UV has to be far from a bottom to avoid the collision with a ground and to avoid the resuspension of bottom water layers. For the automatically choosing of this place, the MM workspace can be presented as a finite numbers of $s$ vertical segments inscribed within [11]. These segments have a parallelepiped shapes with identical bases. Axes of these segments are parallel to the vertical axis $Z$ of the coordinate system $X Y Z$. The value of $s$ is chosen based on size of the MM workspace, soil samplers size and number of samples that you want to take.

The working MM is shown at Fig. 2. Which introduces the next labels: 1 is the bottom; 2 is the bottom surface plane (2); $P_{a}$ is the approach point of the MM sampler 3 (its length is $h$ ); $C_{a}$ is the crossing point of the vertical axis of the segment $a=\overline{1, s}$ with the plane $2 ; 4$ is the MM workspace.

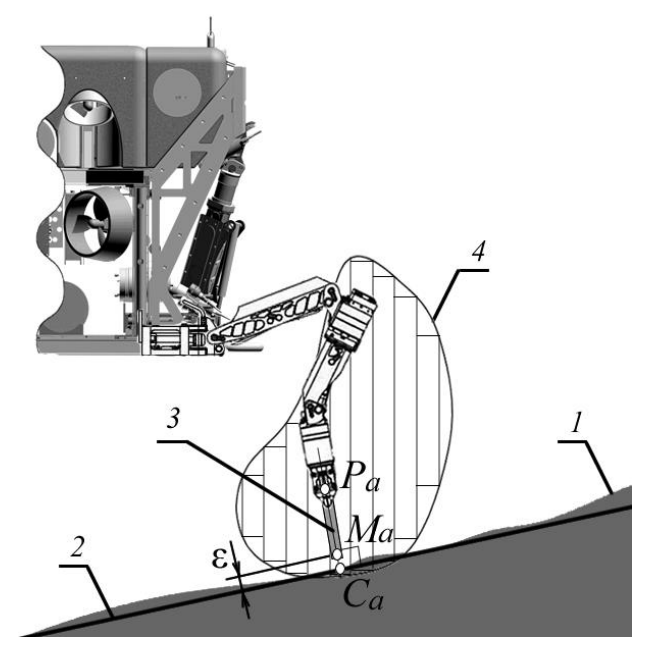

Fig. 2. Performing of the manipulation operation

For every segment $a$ the coordinates $x_{c a}$ and $y_{c a}$ of the crossing point of the segment vertical axis and the plane $X Y$ are known. The coordinate $z_{c a}$ of the crossing point $C_{a}$ is defined by the formula:

$$
z_{c a}=z\left(x_{c a}, y_{c a}\right)=A^{*} x_{c a}+B^{*} y_{c a}+C^{*}
$$

The point $C_{a}\left(x_{c a}, y_{c a}, z_{c a}\right)$ will belong to the MM workspace under the condition:

$$
z_{c a}>z_{c a \min }
$$

where $z_{\text {camin }}$ is the coordinate of the lower base of the segment $a$. Until the point $C_{a}$ satisfying the condition (6) appears, the UV should keep slow submerging to the bottom. When the point $C_{a}$ appears, the UV stops and has to be fixed in this position with help of stabilization systems [5-8].

For the taking of $q$ samples, the UV to dive until the points $C_{a}$ satisfying the condition (6) appear in the $q$ segments. The UV diving stops when $q C_{a}$ points appear. When the UV is stabilized in the hovering mode the MM gripper trajectory can be described as a sequence of transitions from an initial position to the approach point $P_{a}$. Then the gripper transitions by the distance $h$ along the vector $\bar{n}$ to the point $M_{a}$, in which the sampler is maximum immersed. The point $P_{a}$ is formed based on the value $\varepsilon$ (see. Fig. 2). The value $\varepsilon$ allows to eliminate the possible contact of the sampler with the uneven bottom surface in the process of the MM gripper transition from the starting position to the point $P_{a}$ :

$$
P_{a}=C_{a}-\bar{n}(h+\varepsilon)
$$

The point $P_{a}$ must be located in the MM workspace. The coordinates of the point $M_{a}$ are calculated by the formula:

$$
M_{a}=P_{a}+\bar{n} h
$$


For the sampler removing the MM gripper moves to the point $P_{a}$ without changing its orientation. Further, MM moves to the starting position and puts the sampler into a container. It should be noted, that the method [13] allows to perform the manipulation operation even under conditions of the UV unplanned displacements relative to the initial position of its stabilization under the influence of external factors.

\section{Sea tests results}

The sea tests of proposed technology were performed in the Bering Sea with the UV Sub-Atlantic Comanche 18 equipped with the MM Schilling Orion 7P. For this UV the value $L$ is $3.4 \mathrm{~m}$. Coefficients of (2) had been updating with a frequency $5 \mathrm{~Hz}$ of Doppler lag Navigator Teledyne RD Instruments measurements in the process of the UV diving from a distance of $10 \mathrm{~L}$ to a bottom. Fig. 3 shows the bottom surface plane 1 in the coordinate system $X Y Z$. This plane was measured by the lag 2 at the distance of $2 L$ to the sea bottom and described by (2) with the coefficients $A_{n}=0.072$, $B_{n}=0.055, C_{n}=-0.996, p=6.825$. The vector $\bar{n}_{k}$ forms angles $\alpha_{k}=85.9^{\circ}, \beta_{k}=86.8^{\circ}$ and $\gamma_{k}=174.8^{\circ}$ with the respective axes of the coordinate system $X Y Z$.

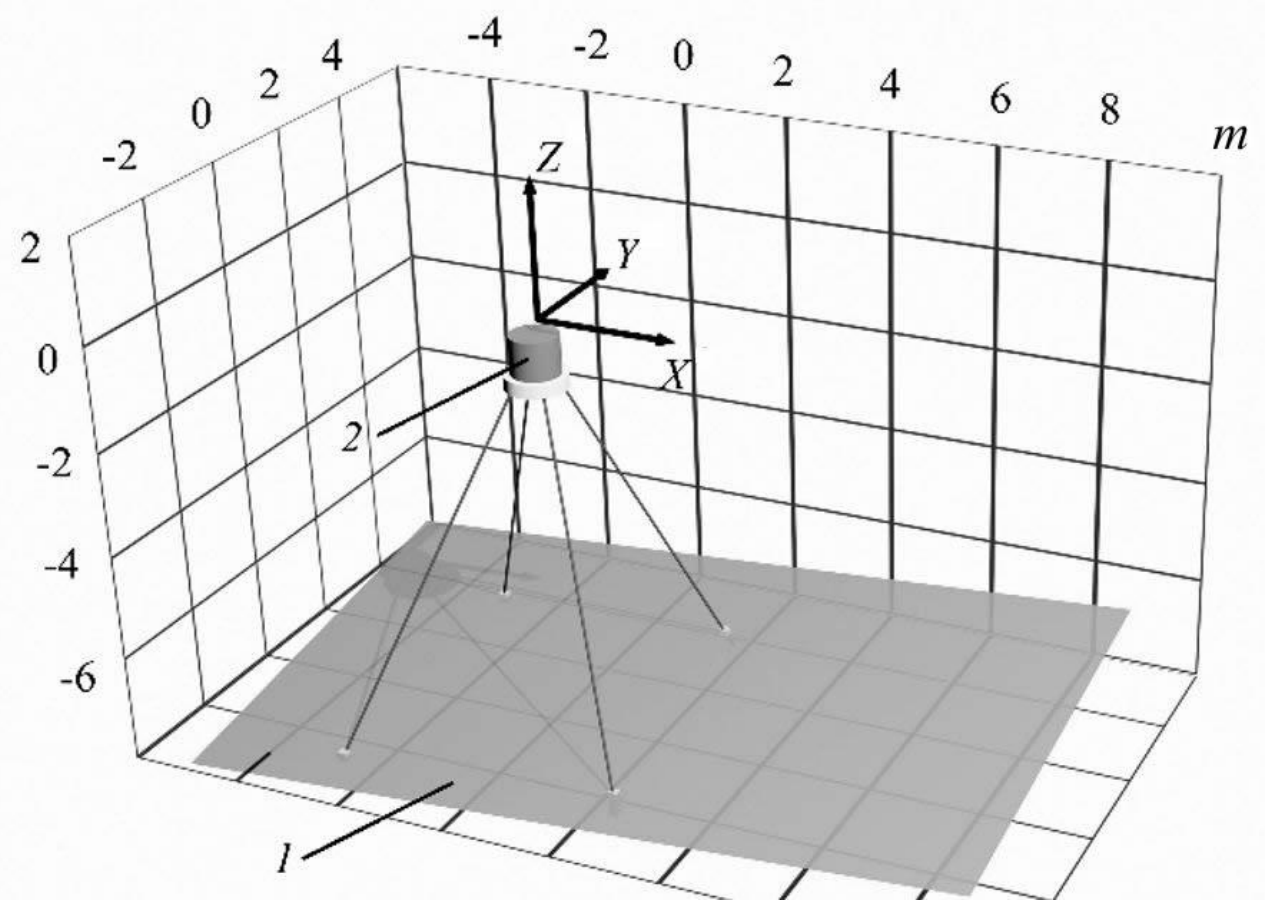

Fig. 3. The results of experimental tests

While the UV was submerged from a distance of $10 L$ to $2 L$ to the bottom, the maximal changes of angles $\alpha_{\mathrm{k}}, \beta_{\mathrm{k}}, \gamma_{\mathrm{k}}$ were less than $5^{\circ}$. Therefore, the conditions (3)-(5) were not violated. The measurements have shown that the bottom surface had no significant irregularities and was suitable for performing of manipulation operations. Few dozen successful dives with the operations of taking of soil samples were performed. In all of these dives the conditions (3)-(5) were always satisfied.

\section{Conclusion}

In this paper, the technology for an automatic implementation of the soil sampling by means of underwater multilink manipulators was considered. This technology provides an automatic performing of the operations of taking soil samples by UV equipped with onboard MM. The position detecting of the sea bottom surface relative to the UV is based on information from a Doppler lag. In the process of UV moving to the bottom, this technology estimates the complexity of the sea bottom relief in the working area and decides on the suitability of this relief for the failsafe performing of desired underwater operations. Also, the algorithm for the automatic performing of operations of taking of soil samples from the bottom surface was proposed. The sea tests were performed for investigation of developed system and algorithm, and confirmed efficiency and simplicity of its practical implementation.

The future studies are aimed to perform more complex manipulation operations by UV in an automatic mode. Some of them are: taking the upper layer of a soil from the surface of the bottom with a complex shape, automatically detecting and taking of underwater objects, as well as performing technological operations, such as cleaning of underwater structures, underwater fouling, welding and carpentry. The technologies for the implementation of said operations will use the information about the object of work will be obtained using multi-beam 3D sonars and technical vision systems. 


\section{Acknowledgments}

This work was performed in Institute of Marine Technology Problems FEB RAS, Institute for Automation and Control Processes FEB RAS and also in Far Eastern Federal University (Vladivostok, Russia).

The development of the technology for the autonomous soil sampling by UV was carried out with the financial support of the Russian Foundation for Basic Research (RFBR) grants (projects 16-29-04195 Ofi_m, 17-57-45055 Ind_a). The development of the method for the estimation of the complexity of a sea bottom relief was carried out with the financial support of the Russian Science Foundation (RSF) grant (project №17-79-10064).

\section{References}

[1] I.E. Tuphanov, A.F. Scherbatyuk. (2012) "Adaptive algorithm of AUV meander pattern trajectory planning for underwater sampling," Proceedings of the 10th ISOPE Pacific/Asia Offshore Mechanics Symposium, PACOMS 2012, Vladivostok, Russia. pp. $181-185$.

[2] V.F. Filaretov, D.A. Yukhimets, A.F. Scherbatyuk, I.E. Tuphanov, E.A. Mursalimov. (2014). "Some Marine Trial Results of a New Method for AUV Trajectory Motion Control," Proceedings of the OCEANS 2014 MTS/IEEE Conference, St. John's, Newfoundland and Labrador, Canada. pp. 1-6.

[3] V.F. Filaretov, A.Yu. Konoplin, N.Yu. Konoplin. (2015). "Method of Synthesis of Automatic Correction Systems of Underwater Vehicles Linear Displacements" Procedia Engineering, Vol 100, pp. 1434-1440.

[4] K. Ishizu, H. Nakayama, N. Sakagami, M. Shibata, S. Kawamura, S. Matsuda, A. Mitsui. (2014). "Preliminary Experiments of a Human-Portable Underwater Gripper Robot for Dexterous Tasks," Proceedings of MTS/IEEE OCEANS 2014.

[5] V.F. Filaretov, A.Yu. Konoplin. (2015). "System of Automatic Stabilization of Underwater Vehicle in Hang Mode with Working Multilink Manipulator," International IEEE Conference on Computer, Control, Informatics and Its Applications (IC3INA), Bandung, Indonesia, pp. 132-137.

[6] V.F. Filaretov, A.Yu. Konoplin. (2016). "Development of Control Systems for Implementation of Manipulative Operations in Hovering Mode of Underwater Vehicle,"/ OCEANS 2016. Shanghai, China. International IEEE Conference.

[7] T.W. McLain, S.M. Rock, M.J. Lee. (1996). "Experiments in the coordinated control of an underwater arm/vehicle system," Autonomous Robots, vol. 3, no. 2-3, pp. 213-232.

[8] M. Santhakumar, K. Jinwhan. (2012). "Indirect adaptive control of an autonomous underwater vehicle-manipulator system for underwater manipulation tasks" Ocean Engineering 54, pp. 233-243.

[9] G. Marani, S.K. Choi, J. Yuh. (2009). "Underwater autonomous manipulation for intervention missions AUVs," Ocean Engineering, vol. 36, no. 1, pp. $15-23$.

[10] D. Lodi Rizzini, F. Kallasi, J. Aleotti, F. Oleari, S. Caselli. (2016) "Integration of a stereo vision system into an autonomous underwater vehicle for pipe manipulation tasks," Computers \& Electrical Engineering, pp. 1-12.

[11] V.F. Filaretov, A.Yu. Konoplin, N.Yu. Konoplin, G.V. Gorbachev. (2016). "Control system for underwater vehicle with multilink manipulator for automatic manipulation operations," Proceedings of the 27th DAAAM International Symposium, B. Katalinic (Ed.), Published by DAAAM International, Vienna, Austria, - pp. 714-720.

[12] G.A. Korn, T.M. Korn. (2000). "Mathematical Handbook for Scientists and Engineers: Definitions, Theorems, and Formulas for Reference and Review," General Publishing Company. - 1151 p.

[13] V.F. Filaretov, A.Yu. Konoplin. (2015). "System of Automatically Correction of Program Trajectory of Motion of Multilink Manipulator Installed on Underwater Vehicle,” Procedia Engineering, Vol. 100, pp. 1441-144. 